\title{
Circulating tumour cells demonstrate an altered response to hypoxia and an aggressive phenotype
}

\author{
K Ameri, ', R Luong ${ }^{2}$, H Zhang', AA Powell', KD Montgomery ${ }^{3}$, I Espinosa ${ }^{3}$, DM Bouley ${ }^{2}$, AL Harris ${ }^{4}$ and \\ SS Jeffrey ${ }^{*, i}$
}

'Department of Surgery Stanford University School of Medicine, Stanford, CA, USA; ${ }^{2}$ Department of Comparative Medicine, Stanford University School of Medicine, Stanford, CA, USA; ${ }^{3}$ Department of Pathology, Stanford University School of Medicine, Stanford, CA, USA; ${ }^{4}$ Department of Medical Oncology and Molecular Oncology Laboratory, Weatherall Institute of Molecular Medicine, University of Oxford, Oxford, UK

\begin{abstract}
BACKGROUND: Tumours contain hypoxic regions that select for an aggressive cell phenotype; tumour hypoxia induces metastasisassociated genes. Treatment refractory patients with metastatic cancer show increased numbers of circulating tumour cells (CTCs), which are also associated with disease progression. The aim of this study was to examine the as yet unknown relationship between hypoxia and CTCs.

METHODS: We generated human MDA-MB-23I orthotopic xenografts and, using a new technology, isolated viable human CTCs from murine blood. The CTCs and parental MDA-MB-23I cells were incubated at 21 and $0.2 \%$ (hypoxia) oxygen, respectively. Colony formation was assayed and levels of hypoxia- and anoxia-inducible factors were measured. Xenografts generated from CTCs and parental cells were compared.

RESULTS: MDA-MB-23I xenografts used to generate CTCs were hypoxic, expressing hypoxia factors: hypoxia-inducible factor I alpha $(\mathrm{HIF|} \mid \alpha)$ and glucose transporter protein type I (GLUTI), and anoxia-induced factors: activating transcription factor 3 and 4 (ATF3 and ATF4). Parental MDA-MB-23I cells induced ATF3 in hypoxia, whereas CTCs expressed it constitutively. Asparagine synthetase (ASNS) expression was also higher in CTCs. Hypoxia induced ATF4 and the HIFI $\alpha$ target gene apelin in CTCs, but not in parental cells. Hypoxia induced lower levels of carbonic anhydrase IX (CAIX), GLUTI and BCL2/adenovirus EIB I9-KD protein-interacting protein 3 (BNIP3) proteins in CTCs than in parental cells, supporting an altered hypoxia response. In chronic hypoxia, CTCs demonstrated greater colony formation than parental cells. Xenografts generated from CTCs were larger and heavier, and metastasised faster than MDA-MB-23I xenografts.

CONCLUSION: CTCs show an altered hypoxia response and an enhanced aggressive phenotype in vitro and in vivo.

British Journal of Cancer (2010) I 02, 56I-569. doi: I0.1038/sj.bjc.660549I www.bjcancer.com

Published online 5 January 2010

(c) 2010 Cancer Research UK
\end{abstract}

Keywords: hypoxia; anoxia; circulating tumour cells; metastasis; xenograft

Metastasis is a multi-step process leading to the spread of neoplastic cells to distant sites. Circulating tumour cells (CTCs) have been detected in the peripheral blood of cancer patients and provide an index of disease aggression in patients with distant metastasis (Dawood et al, 2008). During the metastatic process, CTCs are generated from primary tumour, and subsequently invade and colonise distant organs (Gupta and Massague, 2006). Tumours are heterogeneous and contain regions of hypoxia and anoxia, which may select for cells with more aggressive phenotypes and diminished apoptotic potential (Graeber et al, 1996; Zhang and Hill, 2004). The tumour hypoxic microenvironment contributes to increased metastases by regulating the number of genes

* Correspondence: Dr SS Jeffrey or K Ameri, Department of Surgery, Stanford University School of Medicine, Medical School Lab Surge Building, Room P2 14, I20I Welch Road, Stanford, California, CA 943055494, USA; E-mail: ssj@stanford.edu or kurosh.ameri@gmail.com Received 21 August 2009; revised 23 November 2009; accepted 25 November 2009; published online 5 January 2010 that are implicated in the metastatic process. Clinical evidence also suggests that patients with tumours that contain more extensive hypoxic and anoxic areas have higher rates of metastases (Brizel et al, 1996). Therefore, tumour hypoxia and anoxia may be important in modulating and determining the metastatic ability of CTCs, as well as their progression once secondary tumour metastases are established.

Cells respond to hypoxia and anoxia differently. A major response of cells to hypoxia is the induction of hypoxia-inducible factor 1 (HIF1), which is associated with tumour progression and metastasis (Liao et al, 2007). Hypoxia-inducible factor 1 is a heterodimeric protein that is composed of a constitutively expressed HIF1 $\beta$ subunit and an oxygen $\left(\mathrm{O}_{2}\right)$-regulated HIF1 $\alpha$ subunit. Both the stability and transcriptional activity of HIF1 are negatively regulated by $\mathrm{O}_{2}$-dependent hydroxylation. Under conditions of higher $\mathrm{O}_{2}$ supply, including normoxia, which are used in tissue culture studies $\left(\mathrm{O}_{2}\right.$ levels of $\left.21 \%\right), \mathrm{HIF} 1 \alpha$ is synthesised and hydroxylated on proline residue 402 and/or 564 by prolyl hydroxylase domain proteins that use $\mathrm{O}_{2}$ and $\alpha$-ketoglutarate as substrates. Prolyl hydroxylation is required for the binding 
of the von Hippel-Lindau protein, which then targets HIF $1 \alpha$ for degradation by the proteasome (Epstein et al, 2001). Hypoxiainducible factor $1 \alpha$ is also hydroxylated on asparagine residue 803 by Factor Inhibiting HIF-1 (FIH-1), which negatively regulates the transactivation function (Mahon et al, 2001). With decreasing $\mathrm{O}_{2}$ levels, the rate of HIF $1 \alpha$ hydroxylation is reduced because of substrate $\left(\mathrm{O}_{2}\right)$ deprivation. This results in the accumulation and activation of HIF $1 \alpha$, leading to its dimerisation with HIF1 $\beta$, forming HIF1. Hypoxia-inducible factor 1 then induces target genes that include several adaptive, metastatic, and pro-apoptotic proteins, such as glycolytic enzymes, glucose transporter protein type 1 (GLUT1), lysyl oxidase (LOX), and BCL2/adenovirus E1B $19 \mathrm{KD}$ protein-interactive protein 3 (BNIP3), respectively (Semenza et al, 1996; Sowter et al, 2001; Erler et al, 2006).

The anoxia pathway is distinct from the hypoxia-HIF1 $\alpha$ pathway. Recent studies suggest that extremely low levels of $\mathrm{O}_{2}$, including anoxia, lead to endoplasmic reticulum stress response (ERSR) and activation of a signal transduction pathway known as the unfolded protein response, which is HIF1 $\alpha$ independent. Endoplasmic reticulum stress response triggers a complex programme that results in the reduction of mRNA translation. However, it specifically increases the translation of factor activating transcription factor 4 (ATF4) that protects cells from damaging effects of ER stress (Blais et al, 2004). This same anoxia pathway can also be activated by nutritional deficiencies such as glucose and/or amino acid deprivation, and can lead to ERSR and translational increase of ATF4 (Harding et al, 2000). In addition to an increase in mRNA translation of factors related to the unfolded protein response pathway, such as ATF4, cancer cells also respond to anoxia via other multiple HIF $1 \alpha$-independent pathways, including the mitogen-activated protein kinase pathway and protein stabilisation pathways, which result in the induction of factors such as ATF3 (Ameri et al, 2007; Nemetski and Gardner, 2007) and ATF4 (Ameri et al, 2004; Koditz et al, 2007; Rzymski et al, 2008).

Although the response of cells to hypoxia has been studied in detail and the inter-relationships between hypoxia and anoxia pathways are beginning to emerge, the relationship between CTCs and tumour hypoxia has not been investigated. In humans, triple negative (oestrogen receptor negative, progesterone receptor negative, human epithelial growth factor receptor 2, HER2 negative) breast cancer is an aggressive tumour phenotype that commonly metastasises and for which there is no targeted therapy. The aggression of these tumours may be due to a pronounced hypoxic response (Tan et al, 2009). Using the human triplenegative breast cancer cell line MDA-MB-231, we generated orthotopic xenografts in mice that produced CTCs (Eliane et al, 2008) and resulted in lung metastases. Xenografts were profoundly hypoxic and produced CTCs that could be captured and cultured using a new technology developed by our laboratory. We examined the response of CTCs and parental MDA-MB-231 cells to hypoxia $\left(\mathrm{O}_{2}\right.$ levels of $\left.0.2 \%\right)$ and compared the ability of both cell types to develop tumour xenografts in vivo.

\section{MATERIALS AND METHODS}

\section{Xenograft generation}

NOD.CB17-Prkdc scid/J mice were purchased from The Jackson Laboratory (Bar Harbor, ME and Sacramento, CA, USA). Animals were housed in micro-isolator cages with autoclaved bedding, and fed autoclaved food and water. All experiments were approved by Stanford's Institutional Animal Care and Use Committee and in accordance with all Administrative Panel on Laboratory Animal Care (APLAC) regulations at Stanford University and were in compliance with the National Institutes of Health Guide for Care and Use of Animals. A total of 20 NOD/SCID (non-obese diabetic-severe combined immunodeficiency) female mice had $8 \times 10^{6}$ MDA-MB-231 breast cancer cells in $100 \mu \mathrm{l}$ phosphatebuffered saline (PBS; pH 7.4) plus $100 \mu \mathrm{l}$ of matrigel (BD Biosciences, San Jose, CA, USA) injected into their left second mammary fat pads. Control mice were injected with PBS into the same location. At 55 days after injection, when the average tumour volume was $1.5 \mathrm{~cm}^{3}$, mice were euthanised for isolation of CTCs. To assess the tumour formation capacity of CTCs, mammary fat pads of 10 NOD-SCID mice were injected with a total of $8 \times 10^{6}$ MDA-MB-231 cells or CTCs in a total volume of $200 \mu$, consisting of $100 \mu \mathrm{l}$ PBS and $100 \mu \mathrm{l}$ matrigel. Tumour size was measured over a period of $3-6$ weeks.

\section{Immunohistochemistry}

After the mice were euthanised, their organs and tumours were fixed in formalin, routinely processed, embedded in paraffin, and $5-\mu \mathrm{m}$ sections were placed on glass slides for haematoxylin \& eosin staining and immunohistochemistry (IHC). Microwave heat-induced epitope retrieval in citrate buffer was used for IHC as previously described (Higgins and Warnke, 1999). Tumour and lung sections were stained for the intrinsic marker GLUT1 (ab15309, Abcam, Cambridge, MA, USA), ATF3 (sc-188, Santa Cruz Biotechnology, Santa Cruz, CA, USA), ATF4 (ab31390, Abcam), and HIF1 $\alpha$ (ab51608, Abcam). At $4 \mathrm{~h}$ before being killed, mice were injected through the intraperitoneal route with the hypoxia marker pimonidazole, and tumour and lung sections were stained using Hypoxyprobe-1 (NPI, Belmont, MA, USA).

\section{Isolation of circulating tumour cells}

As soon as the mice were euthanised, blood was drawn by means of a cardiac puncture, recently shown to be the method of choice in obtaining CTCs from mouse blood (Eliane et al, 2008). Mouse blood (200-900 $\mu$ l per mouse) was pooled and collected into a 2-ml EDTA tube (BD, Franklin Lakes, NJ, USA). Blood was diluted to $6 \mathrm{ml}$ with PBS and labelled with magnetic beads functionalised with epithelial cell adhesion molecule antibody (Dynabeads, Invitrogen, Carlsbad, CA, USA). Magnetically labelled blood samples were transferred to a six-well plate and brought up to a final volume of $10 \mathrm{ml}$ using PBS. The magnetically labelled samples were then processed by the newly developed MagSweeper that isolates CTCs by multiple rounds of magnetic capture, wash, and release as previously described (Talasaz et al, 2009). Briefly, magnetic capturing rods covered by plastic sheaths were robotically swept through wells containing magnetically labelled mouse blood. Circulating tumour cells captured on the covered magnetic rods were transferred and washed in a well containing PBS, and then released into another well containing PBS by removal of the magnetic rod from its sheath. After a second cycle of capture, wash, and release, individual CTCs were microscopically visualised and transferred to a new six-well plate containing Dulbecco's modified Eagle's medium high glucose (Invitrogen, Carlsbad, CA, USA) supplemented with $10 \%$ ( $\mathrm{vol} / \mathrm{vol}$ ) foetal calf serum, penicillin $\left(100 \mathrm{U} \mathrm{ml}^{-1}\right)$, and streptomycin $\left(100 \mu \mathrm{g} \mathrm{ml}^{-1}\right)$. The purified CTCs in the six-well plates were cultured in a $5 \% \mathrm{CO}_{2}$ incubator at $37^{\circ} \mathrm{C}$.

\section{Cell culture and hypoxic conditions}

Hypoxia was generated using the anaerobic jar HP0011A (Oxoid, Cambridge, UK and distributed by Remel, Lenexa, KS, USA) and oxygen levels were monitored using the Analox Mini $\mathrm{O}_{2}$ DII monitor (Amron International, Vista, CA, USA). Cells were maintained in Dulbecco's modified Eagle's medium high glucose (Invitrogen) supplemented with $10 \%$ (vol/vol) foetal calf serum, penicillin $\left(100 \mathrm{U} \mathrm{ml}^{-1}\right)$, and streptomycin $\left(100 \mu \mathrm{g} \mathrm{ml}^{-1}\right)$. The 
passage number of CTCs was $<7$. For hypoxia experiments, $3 \times 10^{5}$ of MDA-MB-231 cells or CTCs were seeded in $5-\mathrm{cm}$ plates and incubated with $70-80 \%$ confluency in the humidified jar. A gas mixture of $5 \% \mathrm{CO}_{2} / 95 \% \mathrm{~N}_{2}$ was purged into the jar for $20 \mathrm{~min}$ until the $\mathrm{O}_{2}$ monitor indicated $<0.1 \% \mathrm{O}_{2}$, after which additional gas was purged into the jar for an extra $20 \mathrm{~min}$. When experiments were terminated after $24 \mathrm{~h}$, the $\mathrm{O}_{2}$ monitor indicated oxygen levels of $0.2 \%$.

\section{Colony formation assays}

A total of 400 cells were seeded in 5-cm Petri dishes and incubated for 10 days in either normoxia or hypoxia $(n=5$ for each condition). After 10 days, the colonies were rinsed with PBS and stained with Giemsa stain-modified solution (Sigma-Aldrich, St Louis, MO, USA) for $20 \mathrm{~min}$. Stained colonies were rinsed with water, and visually visible colonies, which consisted of approximately 30 cells, were counted.

\section{Immunoblot}

The ATF3, ATF4 and GLUT1 antibodies used for western analysis were the same as those used for immunostaining. The HIF $1 \alpha$ antibody used for immunoblot analysis was from BD transduction laboratories (610958, BD Biosciences). CAIX (ab15086) and BNIP3 (ab10433) were from Abcam. The apelin antibody (sc-33804) was from Santa Cruz Biotechnology. Cell extracts were generated in a cold room $\left(4^{\circ} \mathrm{C}\right)$. The cell lysis buffer used for preparing total cell extracts was a urea-denaturing buffer $(6.7 \mathrm{M}$ urea, $10 \mathrm{~mm}$ Tris - $\mathrm{HCl}$ ( $\mathrm{pH} 6.8$ ), $5 \mathrm{~mm}$ dithiothreitol, $1 \%$ sodium dodecyl sulphate, and $10 \%$ glycerol) supplemented with Complete mini-protease inhibitor cocktail tablets (Roche Diagnostics, Indianapolis, IN, USA). Cultured cells were washed rapidly once with ice-cold PBS. A volume of $100-200 \mu \mathrm{l}$ of urea lysis buffer was added directly to the plates of cells, scraped into an Eppendorf tube, and sonicated on ice by using a Vibra Cell ultrasonic processor (Sonics\&Materials, Newton, CT, USA). A detergent-compatible bicinchoninic acid protein assay kit (Thermo Scientific, Rockford, IL, USA) was used to estimate the protein concentration of extracts according to the manufacturer's protocol. Total cell extracts (30$60 \mu \mathrm{g}$ per lane) were subjected to reducing sodium dodecyl sulphate-polyacrylamide gel electrophoresis by first mixing equal volumes of protein extract and Laemmli sample buffer containing dithiothreitol and $\beta$-mercaptoethanol and running them on a $12 \%$ readymade gel system (Bio-Rad, Hercules, CA, USA). The resolved proteins were then electroblotted (semidry) onto immune blot polyvinylidene fluoride membrane (Bio-Rad). Bound antibodies were detected using the chemiluminescent substrate ECL + Plus (Amersham Biosciences, Buckinghamshire, UK).

\section{RESULTS}

\section{Hypoxic tumour xenograft model}

Tumour hypoxia and anoxia have been shown to select for an aggressive subtype of tumour cells. Such cells would metastasise to distant organs through the blood stream. We hypothesised that tumours containing regions of hypoxia and anoxia would give rise to CTCs that, on the basis of their selection by hypoxia, should be more aggressive than non-CTCs, and would show an altered response to hypoxia. To examine this, we first generated a tumour model containing profound regions of hypoxia and anoxia. We used the human breast cancer cell line MDA-MB-231 to make orthotopic xenografts and grew them to an average tumour volume of $1.5 \mathrm{~cm}^{3}$. Staining for pimonidazole (Figure 1A) and the intrinsic hypoxia marker GLUT1 (Figure 1B; Airley et al, 2003) confirmed that all tumours contained hypoxic regions, particularly around necrotic areas. Although GLUT1 expression and pimonidazole uptake were more pronounced in tumour cells within perinecrotic
A

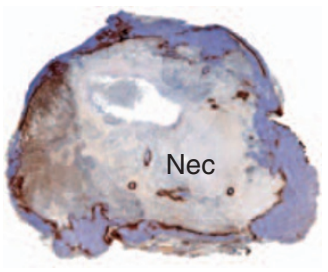

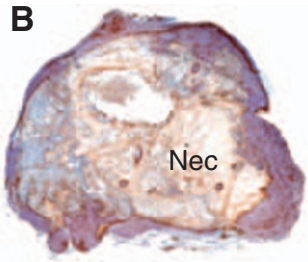

C

i.

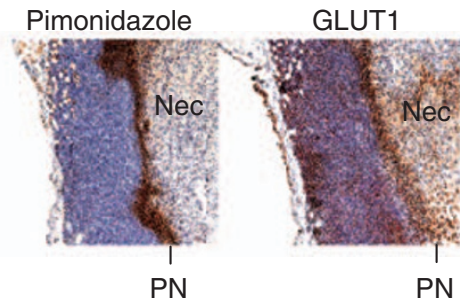

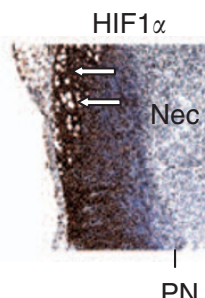

$\mathrm{PN}$
ATF3

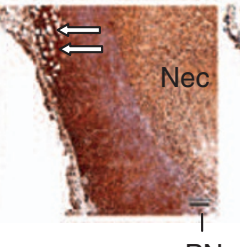

PN

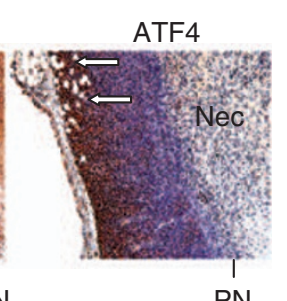

PN

ii.

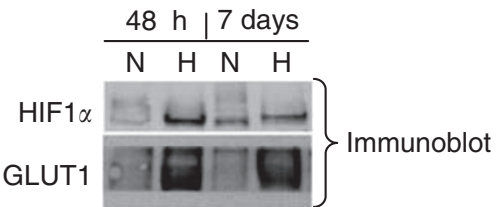

Figure I Human breast cancer MDA-MB-23Icells grown in mammary fat pad of NOD/SCID (Non-obese diabetic-severe combined immunodeficiency) mice display hypoxic/anoxic regions and express hypoxia and anoxia factors. (A) Tumour sections stained for pimonidazole and (B) the intrinsic marker GLUTI ( $\times 10$ magnification). (Ci) HIFI $\alpha$, ATF3, and ATF4 expression in tumours and at the leading edge at sites of local invasion into surrounding normal tissues (white arrows, $\times 100$ magnification). (Cii) Expression of HIFI $\alpha$ and GLUTI in MDA-MB-23। cells after 7 days of chronic hypoxia in vitro. $\mathrm{N}$, normoxia; $\mathrm{H}$, hypoxia; Nec, necrotic region; $\mathrm{PN}$, perinecrotic region. 
regions, the distribution of HIF1 $\alpha$, ATF3, and ATF4 expression rarely coincided with the pimonidazole and GLUT1 staining pattern. (Figure 1Ci).

As GLUT1 is an HIF1 $\alpha$ target gene, one would expect a similar pattern of expression. As tumour cells in perinecrotic regions are more likely to experience chronic hypoxia, we wanted to determine whether MDA-MB-231 cells subjected to chronic hypoxia in vitro would also demonstrate disparate induction of HIF1 $\alpha$ and GLUT1. Whereas HIF $1 \alpha$ and GLUT1 were both induced in acute hypoxia $(48 \mathrm{~h})$, chronic hypoxia (7 days) induced only GLUT1 but not HIF $1 \alpha$ (Figure 1Cii).

Our xenograft model, based on injection of large numbers of tumour cells $\left(8 \times 10^{6}\right)$ and an extended growth period (55 days), created profound regions of hypoxia and anoxia in the primary tumour. Lung metastases developed in all mice. Hypoxia and anoxia factors expressed in primary tumours were also expressed in pulmonary metastases (Figure 2).

\section{Distinctive expression of anoxia-induced factors in hypoxic CTCs}

To determine whether CTCs have an altered hypoxia response, they had to be isolated and grown in culture. We demonstrated that our MDA-MB-231 xenograft model shed CTCs into mouse circulation using a new technology that enabled viable human CTC capture from mouse blood (Talasaz et al, 2009). Magnetic beads attached to the epithelial cell adhesion molecule antigen on CTCs appeared as dark clusters of beads on the cell membrane surrounding translucent cells. In contrast, there were no dark cell/magnetic bead clusters visible in the blood of control mice. Instead, only small dark circles representing individual magnetic beads were observed in control mouse blood (Figure 3Ai). Captured CTCs were viable and could be subsequently grown in culture (Figure 3Aii)
It is known that anoxia exerts a selection pressure for aggressive cells with diminished apoptotic potential. Therefore, anoxiaselected cells in vivo could enter the blood stream as CTCs and demonstrate a distinct expression and/or induction of anoxia factors. To examine this, we compared anoxia factor expression in CTCs with that of parental MDA-MB-231 cells.

The CTCs demonstrated greater expression and/or induction of the anoxia-induced factors ATF3 (Figure 3B), ATF4 (Figure 3C and $\mathrm{D})$, and the ATF4 target gene, ASNS (Figure 3D), than parental MDA-MB-231 cells. Western blot analysis demonstrated that ATF3 protein was expressed constitutively higher in CTCs compared with MDA-MB-231 cells, and hypoxia did not dramatically induce further ATF3 expression as confirmed by densitometry measurements. In contrast, MDA-MB-231 cells induced ATF3 2.4-fold in hypoxia (Figure 3B). Western blot analysis and densitometry measurements demonstrated that the ATF4 protein was not induced in hypoxic MDA-MB 231 cells but was induced 2.4-fold in hypoxic CTCs (Figure 3C). Western blot analysis showed that the ATF4 target gene ASNS was also induced to a greater extent in hypoxic CTCs than in hypoxic MDA-MB-231 cells (Figure 3 D). At a higher cell passage number (passage $>7$ ), CTCs demonstrated some reversion back to the parental phenotype with respect to ATF3 expression and increased induction in hypoxia (data not shown). In summary, CTCs demonstrated an anoxia phenotype by constitutively expressing and/or inducing anoxia factors at higher levels compared with parental MDA-MB 231 cells under normoxia and/or hypoxia.

\section{Distinctive expression of hypoxia-induced factors in CTCs}

In addition to anoxia factor expression, tumour aggression has been related to enhanced expression of hypoxia-induced factors. Therefore, we questioned whether the hypoxic response of CTCs is altered. To examine this, we compared the expression of hypoxia
A

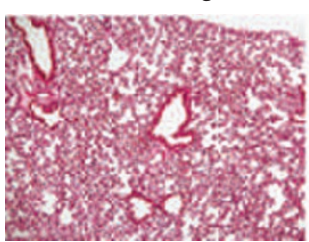

Lung containing tumour metastases

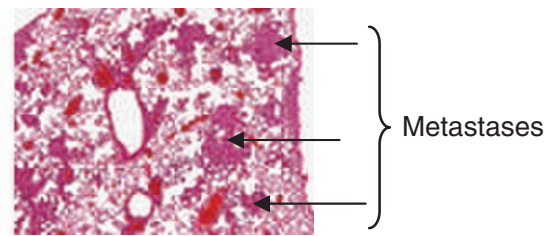

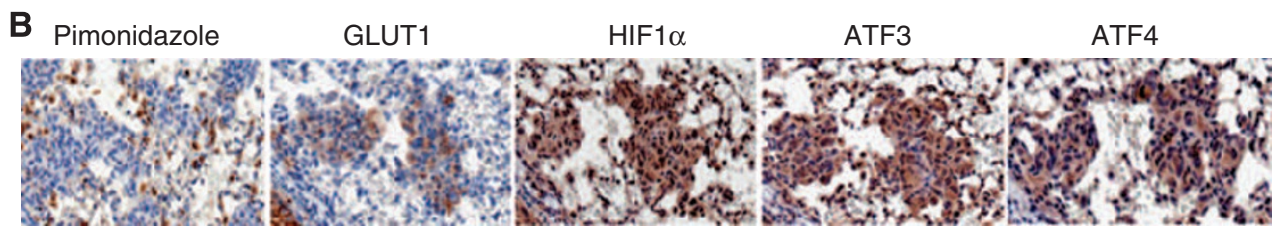

Figure 2 MDA-MB-23I tumours result in pulmonary metastases, which express the hypoxia factors HIF I $\alpha$ and GLUTI, as well as the anoxia factors ATF3 and ATF4. (A) Lungs of xenograft-bearing mice demonstrated metastatic foci, as indicated by the solid arrows on haematoxylin \& eosin (H\&E) slides. Lungs from control mice did not contain any tumours ( $\times 100$ magnification). (B) Expression of HIFI $\alpha$, GLUTI, ATF3, and ATF4 in tumour cells in pulmonary metastatic foci.

Figure 3 Circulating tumour cells (CTCs), isolated and cultured from murine blood, demonstrate a distinct response to hypoxia compared with parental MDA-MB-23I cells. (Ai) Freshly captured CTCs are cells with attached magnetic beads as indicated by white arrows. The magnetic beads are individual small dark circles, as can be seen in the control; CTCs covered with magnetic beads appear as dark clusters of beads on the cell membrane with translucent centres, as indicated by white arrows; lack of CTCs in blood samples obtained from six control mice without xenografts. (Aii) The magnetically captured CTCs in culture on day 10 after capture and after day 60. (B-F) Induction of HIFI $\alpha$ in hypoxic MDA-MB-23I cells and CTCs. (B) Expression of the anoxia factor ATF3 in CTCs and MDA-MB-23I cells. Graphs demonstrate densitometry measurement of ATF3 expression normalised to actin expression from three experiments. (C) Induction of the anoxia-induced factor ATF4 in CTCs compared with MDA-MB-23I cells. Graphs demonstrate densitometry measurements of ATF4 expression normalised to actin expression from three experiments. (D) Expression of ATF4 and its target gene ASNS in CTCs, compared with parental MDA-MB-23I cells. (E and $\mathbf{F}$ ) Differences between CTCs and parental MDA-MB-23I cells with respect to induction of HIFI $\alpha$ target genes. (E) Induction of apelin in hypoxic CTCs compared with parental MDA-MB-23I. Hypoxic induction of (E) GLUTI and (F) CAIX, BNIP3 in CTCs, compared with parental MDA-MB-23I cells. Experiments were performed at least three or four times with three or four different extracts. N, normoxia; $\mathrm{H}$, hypoxia. 


\section{$\underset{O P E N}{B J} \mathrm{O}$}

factors in CTCs and parental MDA-MB-231 cells. Although MDAMB-231 cells and CTCs did not differ in their response to hypoxia with respect to HIF $1 \alpha$ induction, CTCs demonstrated a distinct expression of proteins regulated by HIF1 $\alpha$ under hypoxic conditions. Apelin was induced at greater levels in CTCs than in parental MDA-MB-231 cells (Figure 3E). In hypoxia, CTCs did induce GLUT1 (Figure 3E), CAIX and BNIP3 proteins (Figure 3F) but the inductions were lower than those in parental MDA-MB-231 cells. In summary, CTCs demonstrated an altered response to hypoxia, showing both pronounced (apelin) and reduced induction (GLUT1, CAIX, and BNIP3) of specific hypoxia/HIF1regulated genes.
A

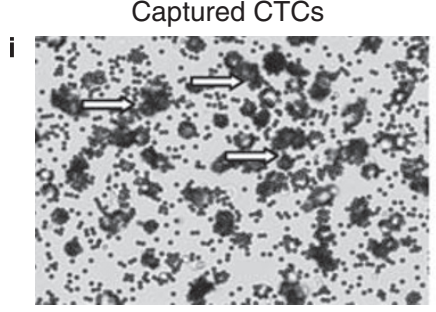

CTC culture day 10

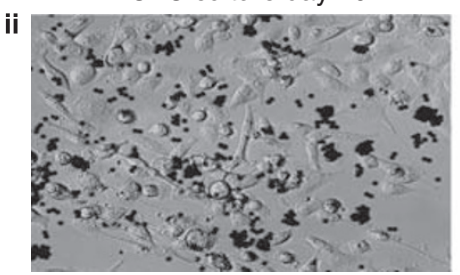

Control
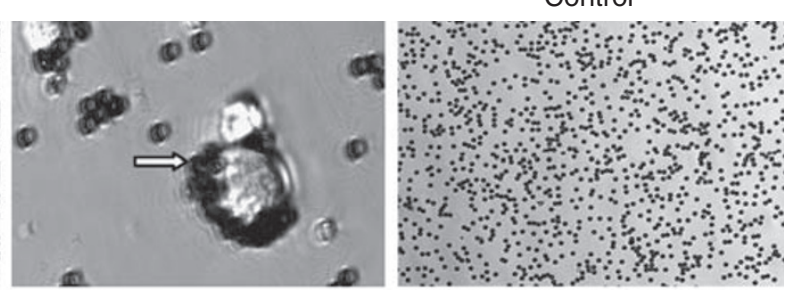

CTC culture day 60

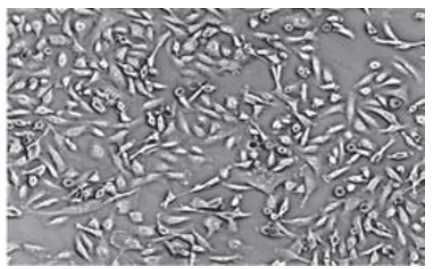

B

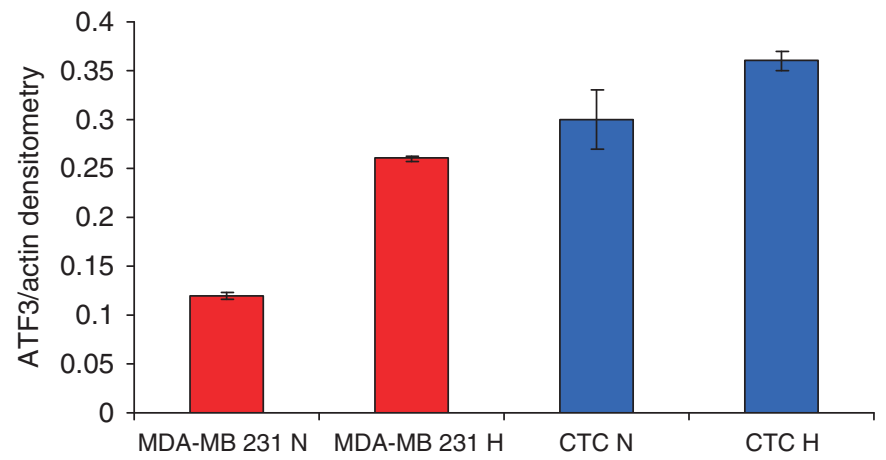

C
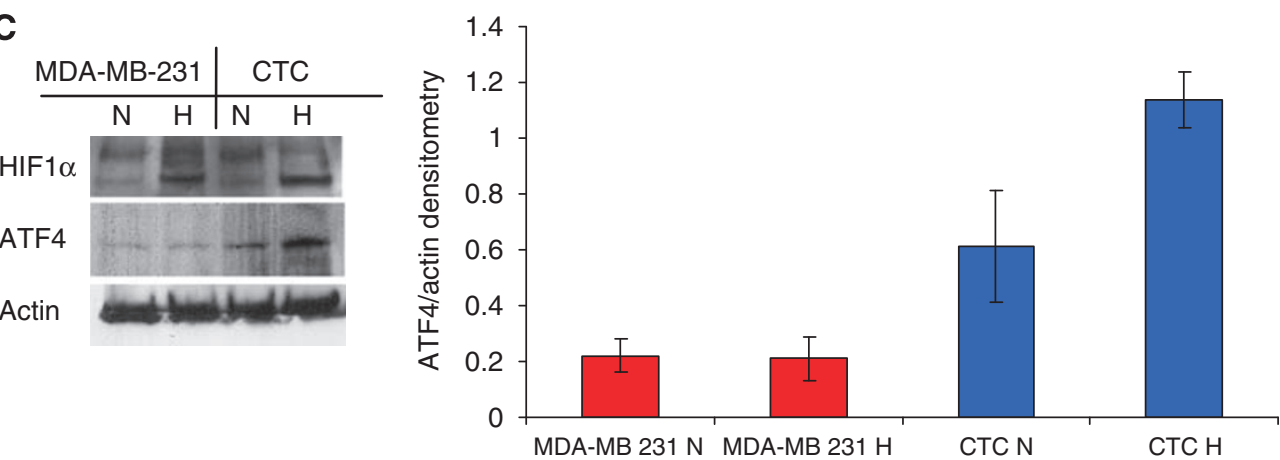

D

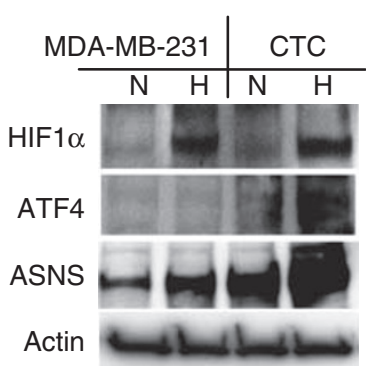

E

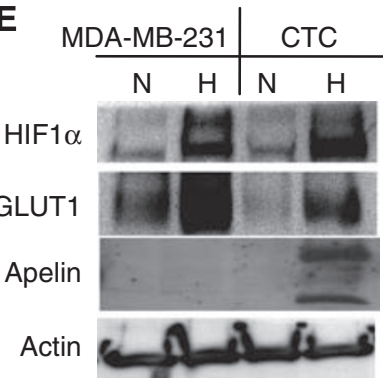

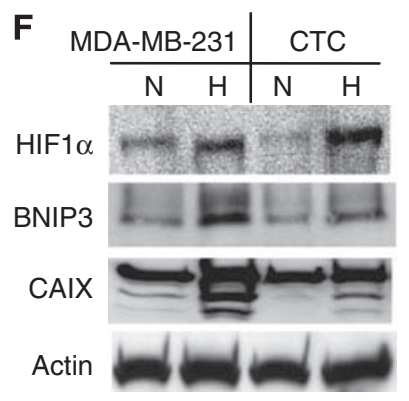


CTCs grow more aggressively as colonies than MDA-MB-231 cells in chronic hypoxia and create larger tumours in vivo, which rapidly result in lung metastasis

The anoxia factors expressed in CTCs are known to be associated with cell proliferation, aggression, and survival. Therefore, we questioned whether CTCs possess an enhanced colony formation capacity in hypoxia. To test this, we performed colony formation assays. A greater number of colonies was observed with CTCs than with parental MDA-MB-231 cells, when grown under conditions of chronic hypoxia for 10 days (Figure 4A). In normoxia, both CTCs and MDA-MB-231 cells resulted in a similar number of colonies after 10 days. Results were similar when different numbers of cells and/or different Petri dish sizes and/or different incubation periods were used for the colony formation assays. Therefore, CTCs had a greater colony formation capacity in chronic hypoxia compared with MDA-MB 231 cells.

The greater clonogenicity under chronic hypoxic conditions suggested that CTCs were more aggressive than their parental MDA-MB-231 cells. To examine tumourigenicity and metastatic potential of CTCs in vivo, we generated 10 orthotopic xenografts from cultured CTCs and compared tumour growth with that of 10 parental MDA-MB-231 xenografts. Within 3 weeks, two mice carrying CTC tumour xenografts died. Of the remaining 8 mice that had CTC xenografts and 10 mice that had MDA-MB-231 xenografts, five were euthanised from each tumour group at week 3. Tumour xenografts in the remaining mice (five with MDA-MB231 xenografts and three with CTC xenografts) continued to grow

A
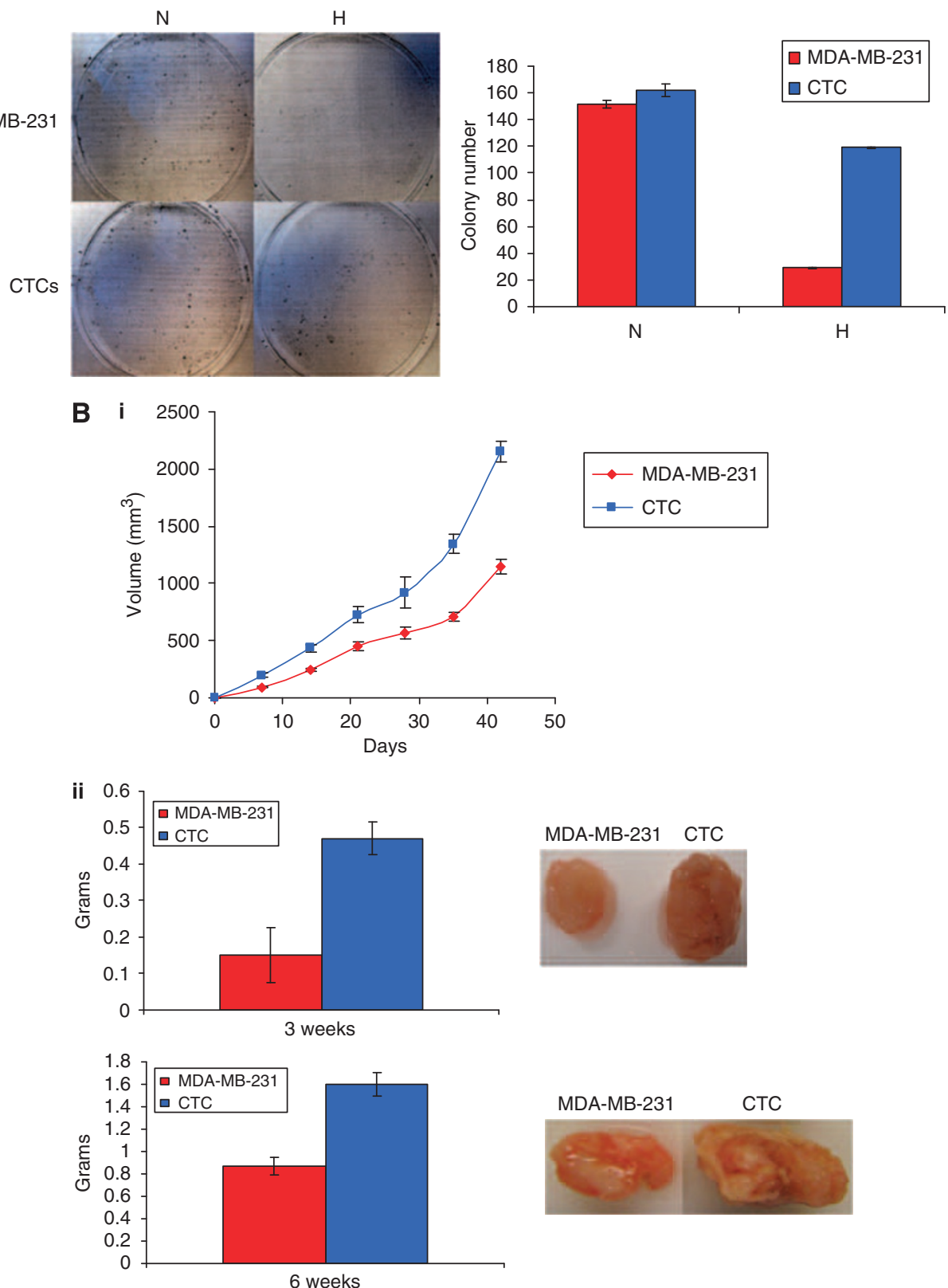

Figure 4 Circulating tumour cells (CTCS) demonstrate greater colony formation capabilities in chronic hypoxia compared with parental MDA-MB-23I cells and grow larger and heavier tumours. (A) Colonies grown from CTCs incubated in normoxia and chronic hypoxia compared with colonies grown from parental MDA-MB-23I cells after 10 days (graph shows mean numbers \pm s.e.). (Bi) Comparing sizes of xenogafts generated from CTCs with xenografts generated from MDA-MB-23I cells within 3-6 weeks of growth (graph represents mean volume \pm s.e., $n=5$ ); (Bii) comparing size and weight of $x$ enografts from CTCs with xenografts from MDA-MB-23I cells at 3 and 6 weeks (graphs represent mean weight \pm s.e.). N, normoxia; H, hypoxia. 
and were measured from week 3 to week 6 , after which the mice were euthanised. As indicated in Figure $4 \mathrm{Bi}$ and ii, xenografts generated from CTCs grew larger and heavier than xenografts from MDA-MB-231 cells. Pulmonary micrometastases became evident at week 3 only in mice with xenografts grown from CTCs. By week 6, mice with parental cell xenografts developed pulmonary metastases. In summary, the more aggressive phenotype of CTCs as observed in vitro by the expression of anoxia factors and greater colony formation in chronic hypoxia was also reflected in vivo by the development of more rapidly growing and metastasising tumours.

\section{DISCUSSION}

Although CTCs have been detected in peripheral blood of cancer patients, and tumours contain areas of hypoxia and anoxia, which is prognostically significant in many clinical studies, the response of CTCs to hypoxia has not been addressed. We have established an approach to generate and isolate viable CTCs to study their response to hypoxia. We did this by first generating xenografts that contained profound areas of hypoxia, and produced lung metastases. Our xenograft model showing more pronounced GLUT1 expression and pimonidazole uptake within perinecrotic regions, and ATF3, ATF4, and HIF1 $\alpha$ expressions throughout the xenografts are in agreement with previous studies of patient tumours showing that GLUT1 expression was mainly perinecrotic and did not coincide with HIF1 $\alpha$ distribution (Sobhanifar et al, 2005; Vleugel et al, 2005; Tan et al, 2009).

Our hypoxic tumour xenograft model shed CTCs into the bloodstream, which we isolated and grew in culture. These CTCs demonstrated an altered response to hypoxia compared with the parental MDA-MB-231 cells from which they were derived. One altered response was ATF4 induction seen only in hypoxic CTCs but not in parental MDA-MB-231 cells. The lack of ATF4 induction in hypoxic MDA-MB-231 cells confirms our previous results in which anoxic conditions rather than hypoxic conditions were shown to be necessary to induce ATF4 (Ameri et al, 2004). The ATF4 target gene ASNS and the anoxia-induced factor ATF3 were also expressed at greater levels in CTCs. These results support the premise that CTCs have a phenotype different than parental MDAMB-231 cells. The reason for such an altered phenotype with respect to expression of anoxia factors remains unclear, but may be because of tumour tissues being hypoxic in vivo and synchronously exposed to nutrition deprivation/ER stress. In this case, the hypoxia pathway alone will not be sufficient for the survival, but additional adaptation responses to nutrition deprivation, such as ATF4-mediated asparagine synthetase (ASNS) induction, are necessary for survival (Cui et al, 2007). Therefore, our results demonstrating that CTCs express the anoxia-induced factors to a much greater extent in hypoxia compared with parental MDA-MB231 cells evoke the possibility that CTCs may have been selected by a combination of tumour hypoxia and nutrition deprivation/ER stress in vivo, and hence, there is greater expression of anoxiainduced and ERSR factors, ATF4 and ASNS, in CTCs.

The mechanism by which CTCs express and/or induce anoxia factors in hypoxic conditions remains to be elucidated. Previous publications have suggested that GLUT1 may modulate the ERSR. For instance, a diminished functional status or lowered expression level of GLUT1 can induce the ERSR arm of the anoxia pathway (Li et al, 2007), and hence result in induction of factors related to the unfolded protein response, such as ATF4. Therefore, it will be important to uncover the potential involvement of a dysregulated hypoxia-HIF1 pathway that could result in regulation of anoxiainduced factors in hypoxic conditions.

Phenotype alterations may be because of the tumour microenvironment, which contains regions of hypoxia, anoxia, and nutritional deficiency that exert evolutionary/selective pressure on tumour cells. Moreover, selected cells that detach from the tumour and become CTCs will be subjected to additional mechanical stress from pressure and shear forces of the circulatory system. Cells that survive such microenvironments may display a more aggressive phenotype. Greater expression of factors: ATF3, ATF4, and ASNS suggests an aggressive CTC phenotype associated with cancer progression and metastasis, and implies a role for HIF1independent pathways in CTCs, which could be considered for future therapeutic interventions. Indeed, our results showed that CTCs were more aggressive in vitro under chronic hypoxia and in vivo, which may be due to more pronounced anoxia pathways. The ATF3 is a gene with a known role in metastasis, and has been described to induce genes relevant to epithelial-to-mesenchymal transition (Yin et al, 2008), a histological feature of invading cells (Guarino et al, 2007). Activating transcription factor 4 is involved in drug resistance (Igarashi et $a l, 2007$ ) and is a major factor in nutrition sensing, regulating the expression of ASNS (Siu et al, 2002). Under conditions of hypoxia and/or glucose deprivation, cells can switch to ATF4-mediated ASNS expression as a protective mechanism (Cui et al, 2007). Furthermore, ASNS is also known to be associated with drug resistance in leukaemia (Williams, 2007) and oncogenesis triggered by mutated p53 (Scian et al, 2004).

In addition to expressing the anoxia-induced factors at greater levels, CTCs also demonstrated an altered regulation of hypoxiaHIF $1 \alpha$ target genes. Circulating tumour cells did induce BNIP3, CAIX, and GLUT1, but the induction of these factors was much lower than in parental MDA-MB-231 cells. In contrast, induction of apelin was much higher in CTCs, compared with parental MDAMB-231 cells. These results further support that CTCs have an altered phenotype. The distinctive expression of hypoxia-induced factors could have implications in the outcome of CTC-mediated metastasis and in secondary tumour survival and growth. The significance of greater induction of apelin could be relevant to tumour growth and metastasis. Apelin is a potent activator of tumour angiogenesis and is associated with earlier onset of tumour development and increased tumour growth in vivo (Sorli et al, 2007). The consequences of decreased BNIP3 induction in CTCs may determine the metastatic potential, as the expression of BNIP3 has been associated with a less-aggressive phenotype in invasive breast carcinoma (Tan et al, 2007), and a reduction in expression of BNIP3 has been suggested to be critical for metastasis (Manka et al, 2005).

The mechanism that accounts for the distinct hypoxia/HIF1 response in CTCs is a subject for further investigation. Activating transcription factor 4 may downregulate certain HIF $1 \alpha$ genes but upregulate others (Rzymski et al, 2007), and during completion of this study, it was shown that ATF4 can modulate the hypoxic induction of HIF1 target genes selectively by inducing CAIX but not GLUT1 in mouse embryonic fibroblasts, as well as in the human colon cancer cell line HCT116 and glioblastoma cell line U373 (van den Beucken et al, 2009). In contrast, we did not observe greater induction of CAIX in CTCs in which ATF3 and ATF4 were highly induced by hypoxia. Activating transcription factor 4 (Ameri and Harris, 2008) and ATF3 (Hai et al, 1999) are known to be transcriptional activators and repressors, and ATF3 is known to antagonise ATF4. Thus, whether ATF3 antagonises ATF4-mediated induction of CAIX in CTCs needs to be considered. In addition, regulation of CAIX by ATF4 could be cancer-type specific.

Cross-sectional evaluation of CTC tumours did not show smaller zones of necrosis that might be expected if all CTCs are assumed to be resistant to hypoxia. We think that areas of necrosis seen in CTC tumours are due to greater proliferation in hypoxia, with a rapid enlargement of tumour mass outgrowing blood/nutrient supply, and further selection of aggressive cells within a CTC tumour. If such highly metabolic cells consumed residual oxygen and nutrients, they would deprive less aggressive populations of critical nutrients and promote their death. We observed that with higher passage numbers 
of CTCs in culture, there was some reversion back to parental phenotype. If some CTC daughter cell populations growing in vivo also reverted to parental cell behaviour during subcutaneous tumour growth, these tumour cells could be less resistant to hypoxia and could affect necrotic zone size.

In summary, we demonstrate that CTCs show an altered phenotype compared with the parental tumour cells from which they were derived. We show that CTCs demonstrate a distinct response to hypoxia in vitro and a greater aggression in vivo. Our study also shows that the circulating cells captured from blood by the MagSweeper are indeed cancer cells in that they produce tumours in mice that metastasise. Our experimental system may be applied to test drug efficacy in targeting CTCs for the prevention of metastases as suggested by Bondareva et al (2009). Our approach and data provide a foundation to investigate selective factors associated with CTC aggressiveness and enable the identification of additional therapeutic targets related to tumour hypoxia and CTCs.

\section{REFERENCES}

Airley RE, Loncaster J, Raleigh JA, Harris AL, Davidson SE, Hunter RD, West CM, Stratford IJ (2003) GLUT-1 and CAIX as intrinsic markers of hypoxia in carcinoma of the cervix: relationship to pimonidazole binding. Int J Cancer 104: 85-91

Ameri K, Hammond EM, Culmsee C, Raida M, Katschinski DM, Wenger RH, Wagner E, Davis RJ, Hai T, Denko N, Harris AL (2007) Induction of activating transcription factor 3 by anoxia is independent of p53 and the hypoxic HIF signalling pathway. Oncogene 26: 284-289

Ameri K, Harris AL (2008) Activating transcription factor 4. Int J Biochem Cell Biol 40: $14-21$

Ameri K, Lewis CE, Raida M, Sowter H, Hai T, Harris AL (2004) Anoxic induction of ATF-4 through HIF-1-independent pathways of protein stabilization in human cancer cells. Blood 103: $1876-1882$

Blais JD, Filipenko V, Bi M, Harding HP, Ron D, Koumenis C, Wouters BG, Bell JC (2004) Activating transcription factor 4 is translationally regulated by hypoxic stress. Mol Cell Biol 24: 7469-7482

Bondareva A, Downey CM, Ayres F, Liu W, Boyd SK, Hallgrimsson B, Jirik FR (2009) The lysyl oxidase inhibitor, beta-aminopropionitrile, diminishes the metastatic colonization potential of circulating breast cancer cells. PLoS One 4: e5620

Brizel DM, Scully SP, Harrelson JM, Layfield LJ, Bean JM, Prosnitz LR, Dewhirst MW (1996) Tumor oxygenation predicts for the likelihood of distant metastases in human soft tissue sarcoma. Cancer Res 56: 941 - 943

Cui H, Darmanin S, Natsuisaka M, Kondo T, Asaka M, Shindoh M, Higashino F, Hamuro J, Okada F, Kobayashi M, Nakagawa K, Koide H (2007) Enhanced expression of asparagine synthetase under glucose-deprived conditions protects pancreatic cancer cells from apoptosis induced by glucose deprivation and cisplatin. Cancer Res 67: $3345-3355$

Dawood S, Broglio K, Valero V, Reuben J, Handy B, Islam R, Jackson S, Hortobagyi GN, Fritsche H, Cristofanilli M (2008) Circulating tumor cells in metastatic breast cancer: from prognostic stratification to modification of the staging system? Cancer 113: 2422-2430

Eliane JP, Repollet M, Luker KE, Brown M, Rae JM, Dontu G, Schott AF, Wicha M, Doyle GV, Hayes DF, Luker GD (2008) Monitoring serial changes in circulating human breast cancer cells in murine xenograft models. Cancer Res 68: 5529-5532

Epstein AC, Gleadle JM, McNeill LA, Hewitson KS, O’Rourke J, Mole DR, Mukherji M, Metzen E, Wilson MI, Dhanda A, Tian YM, Masson N, Hamilton DL, Jaakkola P, Barstead R, Hodgkin J, Maxwell PH, Pugh CW, Schofield CJ, Ratcliffe PJ (2001) C. elegans EGL-9 and mammalian homologs define a family of dioxygenases that regulate HIF by prolyl hydroxylation. Cell 107: $43-54$

Erler JT, Bennewith KL, Nicolau M, Dornhofer N, Kong C, Le QT, Chi JT, Jeffrey SS, Giaccia AJ (2006) Lysyl oxidase is essential for hypoxiainduced metastasis. Nature 440: $1222-1226$

Graeber TG, Osmanian C, Jacks T, Housman DE, Koch CJ, Lowe SW, Giaccia AJ (1996) Hypoxia-mediated selection of cells with diminished apoptotic potential in solid tumours. Nature 379: $88-91$

\section{ACKNOWLEDGEMENTS}

This study was supported in part by National Institutes of Health grant R01GM085601 (SSJ), the California Breast Cancer Research Program of the University of California, Grant Number 11IB-0175 (SSJ), the John and Marva Warnock Cancer Research Fund, and the Rachleff family fund. KA was supported by fellowship funds from Bernard and Susan Liautaud and the Stanford University School of Medicine Dean's Scholar Award.

\section{Conflict of interest}

Drs Stefanie Jeffrey and Ashley Powell are co-inventors of the MagSweeper CTC isolation technology used in this study. They can receive royalties from this invention through Stanford, but Dr Jeffrey has donated her royalties to support student programmes at The Jackson Laboratory, a non-profit biomedical research institution.
Guarino M, Rubino B, Ballabio G (2007) The role of epithelialmesenchymal transition in cancer pathology. Pathology 39: 305-318

Gupta GP, Massague J (2006) Cancer metastasis: building a framework. Cell 127: $679-695$

Hai T, Wolfgang CD, Marsee DK, Allen AE, Sivaprasad U (1999) ATF3 and stress responses. Gene Expr 7: $321-335$

Harding HP, Novoa I, Zhang Y, Zeng H, Wek R, Schapira M, Ron D (2000) Regulated translation initiation controls stress-induced gene expression in mammalian cells. Mol Cell 6: $1099-1108$

Higgins JP, Warnke RA (1999) CD30 expression is common in mediastinal large B-cell lymphoma. Am J Clin Pathol 112: 241 - 247

Igarashi T, Izumi H, Uchiumi T, Nishio K, Arao T, Tanabe M, Uramoto H, Sugio K, Yasumoto K, Sasaguri Y, Wang KY, Otsuji Y, Kohno K (2007) Clock and ATF4 transcription system regulates drug resistance in human cancer cell lines. Oncogene 26: 4749 - 4760

Koditz J, Nesper J, Wottawa M, Stiehl DP, Camenisch G, Franke C, Myllyharju J, Wenger RH, Katschinski DM (2007) Oxygen-dependent ATF-4 stability is mediated by the PHD3 oxygen sensor. Blood 110: $3610-3617$

Li J, Wang XM, Wang Q, Yang M, Feng XC, Shen ZH (2007) Downregulation of $\mathrm{N}$-acetylglucosaminyltransferase-V induces ER stress by changing glycosylation and function of GLUT1. Arch Biochem Biophys 463: $102-109$

Liao D, Corle C, Seagroves TN, Johnson RS (2007) Hypoxia-inducible factor-1alpha is a key regulator of metastasis in a transgenic model of cancer initiation and progression. Cancer Res 67: 563-572

Mahon PC, Hirota K, Semenza GL (2001) FIH-1: a novel protein that interacts with HIF-1alpha and VHL to mediate repression of HIF-1 transcriptional activity. Genes Dev 15: $2675-2686$

Manka D, Spicer Z, Millhorn DE (2005) Bcl-2/adenovirus E1B 19-kDa interacting protein-3 knockdown enables growth of breast cancer metastases in the lung, liver, and bone. Cancer Res 65: 11689-11693

Nemetski SM, Gardner LB (2007) Hypoxic regulation of Id-1 and activation of the unfolded protein response are aberrant in neuroblastoma. J Biol Chem 282: $240-248$

Rzymski T, Paantjens A, Bod J, Harris AL (2007) American Association Cancer Research, AACR. Abstract Number 4596

Rzymski T, Paantjens A, Bod J, Harris AL (2008) Multiple pathways are involved in the anoxia response of SKIP3 including HuR-regulated RNA stability, NF-kappaB and ATF4. Oncogene 27: 4532-4543

Scian MJ, Stagliano KE, Deb D, Ellis MA, Carchman EH, Das A, Valerie K, Deb SP, Deb S (2004) Tumor-derived p53 mutants induce oncogenesis by transactivating growth-promoting genes. Oncogene 23: 4430-4443

Semenza GL, Jiang BH, Leung SW, Passantino R, Concordet JP, Maire P, Giallongo A (1996) Hypoxia response elements in the aldolase A, enolase 1 , and lactate dehydrogenase A gene promoters contain essential binding sites for hypoxia-inducible factor 1. J Biol Chem 271: 32529-32537

Siu F, Bain PJ, LeBlanc-Chaffin R, Chen H, Kilberg MS (2002) ATF4 is a mediator of the nutrient-sensing response pathway that activates the human asparagine synthetase gene. J Biol Chem 277: 24120-24127 
BJC $\cap$

Sobhanifar S, Aquino-Parsons C, Stanbridge EJ, Olive P (2005) Reduced expression of hypoxia-inducible factor-1alpha in perinecrotic regions of solid tumors. Cancer Res 65: 7259-7266

Sorli SC, Le Gonidec S, Knibiehler B, Audigier Y (2007) Apelin is a potent activator of tumour neoangiogenesis. Oncogene 26: 7692-7699

Sowter HM, Ratcliffe PJ, Watson P, Greenberg AH, Harris AL (2001) HIF-1dependent regulation of hypoxic induction of the cell death factors BNIP3 and NIX in human tumors. Cancer Res 61: 6669-6673

Talasaz AH, Powell AA, Huber DE, Berbee JG, Roh KH, Yu W, Xiao W, Davis MM, Pease RF, Mindrinos MN, Jeffrey SS, Davis RW (2009) Isolating highly enriched populations of circulating epithelial cells and other rare cells from blood using a magnetic sweeper device. Proc Natl Acad Sci USA 106: $3970-3975$

Tan EY, Campo L, Han C, Turley H, Pezzella F, Gatter KC, Harris AL, Fox SB (2007) BNIP3 as a progression marker in primary human breast cancer; opposing functions in in situ versus invasive cancer. Clin Cancer Res 13: $467-474$

Tan EY, Yan M, Campo L, Han C, Takano E, Turley H, Candiloro I, Pezzella F, Gatter KC, Millar EK, O'Toole SA, McNeil CM, Crea P, Segara D, Sutherland RL, Harris AL, Fox SB (2009) The key hypoxia regulated gene CAIX is upregulated in basal-like breast tumours and is associated with resistance to chemotherapy. $\mathrm{Br} J$ Cancer 100: $405-411$

van den Beucken T, Koritzinsky M, Niessen H, Dubois L, Savelkouls K, Mujcic H, Jutten B, Kopacek J, Pastorekova S, van der Kogel AJ, Lambin P, Voncken W, Rouschop KM, Wouters BG (2009) Hypoxia-induced expression of carbonic anhydrase 9 is dependent on the unfolded protein response. J Biol Chem 284: 24204-24212

Vleugel MM, Greijer AE, Shvarts A, van der Groep P, van Berkel M, Aarbodem Y, van Tinteren H, Harris AL, van Diest PJ, van der Wall E (2005) Differential prognostic impact of hypoxia induced and diffuse HIF-1alpha expression in invasive breast cancer. J Clin Pathol 58: $172-177$

Williams DA (2007) A new mechanism of leukemia drug resistance? N Engl J Med 357: $77-78$

Yin X, Dewille JW, Hai T (2008) A potential dichotomous role of ATF3, an adaptive-response gene, in cancer development. Oncogene 27: 2118-2127

Zhang L, Hill RP (2004) Hypoxia enhances metastatic efficiency by upregulating $\mathrm{Mdm} 2$ in KHT cells and increasing resistance to apoptosis. Cancer Res 64: 4180-4189

(c) (1) This work is licensed under the Creative Commons c) BY NC SA Attribution-NonCommercial-Share Alike 3.0 License. To view a copy of this license, visit http://creativecommons.org/ licenses/by-nc-sa/3.0/ 